\title{
High-Resolution InSAR Building Layovers Detection and Exploitation
}

\author{
Cristian Rossi and Michael Eineder, Senior Member, IEEE
}

\begin{abstract}
Layover affects the quality of urban interferometric synthetic aperture radar (InSAR) digital elevation models. Moreover, it is generally difficult to interpret because of the superposition of several contributions in a single SAR pixel. In this paper, a novel technique for the extraction of building layovers is first presented. It makes use of the geocoding stage embedded in the InSAR processor. It is shown that building layovers create a regular pattern in the mapping counter, a map describing the number of occurrences of a SAR pixel in the elevation model. Its exploitation yields a generation of a layover map without the use of external supports. The integration in the processor with a limited additional computational load and the capability to isolate layover signatures are additional benefits. Layover patches are then individually analyzed toward a better understanding of the complex urban signal return. A spectral estimation framework is employed to assess the slopes superimposed in the patches. Fringe-frequency estimation is involved. A set of simulations made for a nonparametric (fast Fourier transform) and a parametric (multiple signal classification) technique is performed prior to testing on real data. It is demonstrated that in X-band, for a single interferogram, just one layover contributor, when it dominates over the others, can be extracted with a sufficient accuracy. The algorithms are tested on a TanDEM-X spotlight acquisition over Berlin (Germany).
\end{abstract}

Index Terms-Fringe-frequency estimation, geocoding, interferometric SAR (InSAR), layover detection, layover scattering decomposition, super-resolution, urban mapping.

\section{INTRODUCTION}

A N URBAN area is probably the most complex terrain to map with synthetic aperture radar (SAR) sensors. Geometrical distortions such as layover and shadow [1] always occur due to the SAR side looking viewing geometry on the frequent metropolitan slope discontinuities. Besides distortions, multiple scattering phenomena and building overlays make data interpretation difficult [2]-[4]. In this context, a height map generation is quite a challenging issue. Optical and LiDAR remote sensing technologies are widely used for this purpose [5], [6]. Next to them, urban reconstruction with SAR data is attractive, considering the increasing number of civil missions. For instance, inherent sensor proprieties such as night and atmosphere-free vision and data globalization may overcome optical and LiDAR limitations. In general, a surface model is a demanding product for many management applications and

Manuscript received October 2, 2014; revised February 20, 2014; accepted May 30, 2015.

The authors are with the Remote Sensing Technology Institute, German Aerospace Center (DLR), D-82234 Oberpfaffenhofen, Germany (e-mail: cristian.rossi@dlr.de; michael.eineder@dlr.de).

Color versions of one or more of the figures in this paper are available online at http://ieeexplore.ieee.org.

Digital Object Identifier 10.1109/TGRS.2015.2440913 several studies have been reported in the recent years employing interferometric SAR (InSAR). They can be grouped in two research branches. Multibaselines techniques use a stack of SAR images over a specific area to derive the elevation information. Contrariwise, single-baseline techniques exploit the sole interferometric phase generated with two acquisitions. Within the first branch, tomographic algorithms have been applied to SAR (TomoSAR) with reconstruction accuracy depending on the number of images that has been used, the signal-tonoise ratio (SNR), and the baseline distribution [7], [8]. In the interesting parameter range of TomoSAR, the achievable height accuracy is on the order of a meter. In the tomographic approach, layover patches are not predetected, but the number of layover components are estimated in a pixel-wise fashion for a set of candidate pixels [9]. Within the second branch, in [10] a stochastic framework has been established to jointly retrieve a building classification and a height map. A mean root-mean-square error (RMSE) of $2.5 \mathrm{~m}$ has been reported for 19 buildings. Layover is estimated from the surface model itself and used to correct the classification map, but not the layover height. First studies about large-scale single-baseline polarimetric InSAR (PolInSAR) building height estimation have been presented in [11]. RMSE over 140 reference building was reported to be around $3 \mathrm{~m}$. In [12], the TanDEM-X mission, in a single-baseline configuration, has been stated capable to generate a high-resolution surface model on a raster of $2.5 \mathrm{~m}$ over urban areas with a RMSE of about $8 \mathrm{~m}$ for the complete model. In addition, here, layover elevations are neither detected nor corrected. In short, the single-baseline reconstruction accuracy is severely limited in layover zones, where multiple facets are mapped in a single SAR resolution cell. For this reason, a precise identification of those areas is of fundamental importance for a proper digital elevation model (DEM) quality assessment. Additional applications for which the layover map is a useful support are urban object simulation, detection and analysis [13], [14] and change detection aimed at disaster management [15].

Precise layover detection usually requires an accurate input DEM. In particular, the elevation model has to be reprojected in SAR coordinates (slant range, azimuth) and the distance between satellite and model cell to be computed. A change of sign in the derivative of the distance function for iso-azimuth lines identifies the beginning of the layover area [16]. With this method, a right layover map can be generated for urban areas only when a sufficiently accurate DEM over the area is available (e.g., a LiDAR one). To be noticed, the DEM generated employing one SAR interferogram cannot be used for the purpose as not accurate enough on building layover positions 
[12]. As an alternative, without a reference DEM, the particular layover phase trend [17] can be exploited. Preliminary studies on the layover detection exploiting the interferometric singlebaseline wrapped phase have been performed [18]. In this paper, a novel technique to detect building layovers is introduced. The InSAR layover model is described in Section II. Layover geocoding, i.e., how layover pixels appear in the generated DEM, is analyzed in Section III. These two sections provide the theoretical background for the detection method presented in Section IV. The absence of building model hypothesis and the low computational cost are considerable aspects of the proposed algorithm.

The second part of this paper deals with the exploitation of the detected patches. In practice, building layover is composed of several contributors. At least two contributors superimpose in the layover area. Each contributor lies on a terrain slope (e.g., ground slope, wall slope, or roof slope). Once layover patches are detected, slope estimation can be carried out through a frequency analysis, reminding that the interferometric phase is proportional to terrain slopes [19]. In the SAR community, the fringe-frequency estimation is a well-known topic. The single frequency estimation is often linked to the phase unwrapping problem [20], whereas the multiple estimation to the tomographic framework [7]. As aforementioned, the latter case exploits a multibaseline data stack to provide the layover decomposition. In this paper, the estimation is instead performed in the spatial domain, looking for the number of slopes included in a building layover patch. In Section V, this problem is studied in detail with simulations and tested on real data. The focus is on the particular urban case, considering the periodogram and the conventional multiple signal classification (MUSIC) algorithm as references.

To introduce the problem, a clear example of single layover contributor dominance is shown in Fig. 1, a high-resolution spotlight interferometric data take [21] over Las Vegas, USA, acquired with TanDEM-X. In Fig. 1(a), the master channel amplitude is shown with the slant range coordinate in vertical direction, to highlight skyscraper layovers with a human-eye perspective. These high-rise constructions are well visible in the SAR amplitude as their layover signal is mainly dominated by their facades. Due to the typical skyscraper structure, i.e., extending in the vertical more than in the horizontal dimension, at the side-looking SAR geometry, roof features are superimposed just in a small layover portion and not in the full layover area. Closely inspecting the layover return, it is possible to detect structural features that behave as corner reflectors (e.g., window eaves). These features have a stronger backscatter than ground scatterers, thus making facade layover contributors dominate over others. The impact in the TanDEM-X interferogram is represented in Fig. 1(b). At skyscraper locations, a regular fringe pattern is well visible. The range fringe frequency corresponds to a vertical terrain, meaning that layover range cells are fully characterized by scatterers at the building facade, despite the superposition with ground scatterers. By counting the number of fringes and considering the height of ambiguity of $33 \mathrm{~m}$, it is even possible to provide an estimate of the local skyscraper height. Whereas for high-rise buildings, layover detection seems to be at least a visually manageable task,

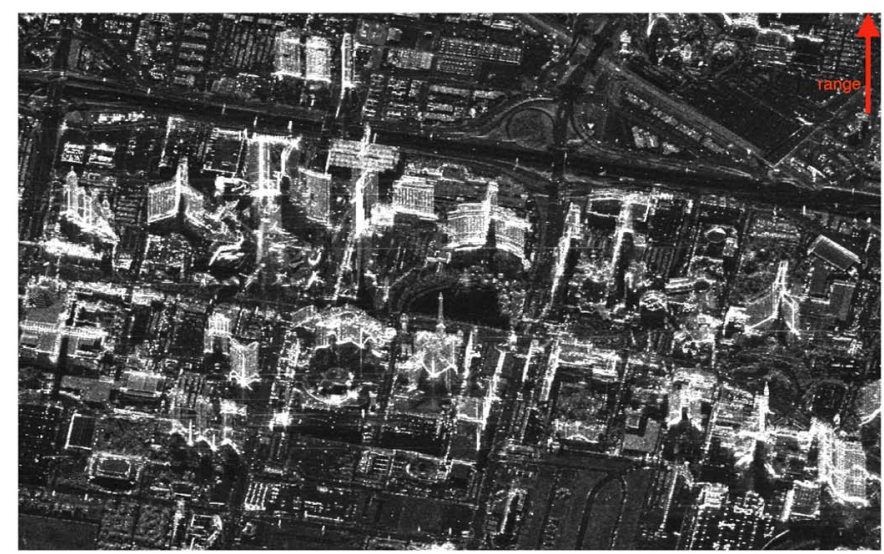

(a) master channel amplitude

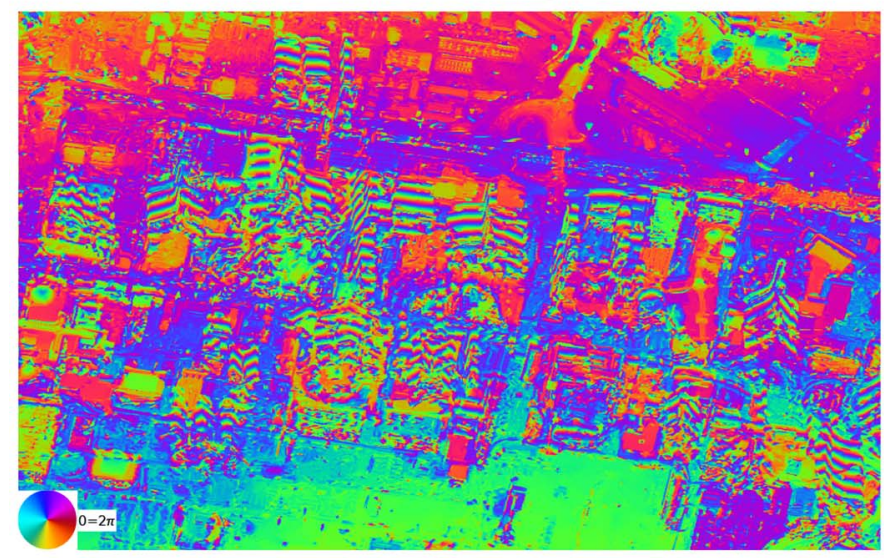

(b) flattened interferogram

Fig. 1. Las Vegas The Strip as imaged by a spotlight TanDEM-X take. (a) Skyscraper layovers show a facade dominance in the amplitude signal. (b) The flattened interferogram at the skyscraper locations presents a regular fringe pattern.

this is not the case for regular buildings. Mixed contributions do not provide a clear fringe pattern. Rooftops may be fully included in the layover patch depending on the incidence angle, and structures as chimneys and antennas mix up with facade structures yielding a difficult interpretation. Tilted roof slopes may superimpose with the vertical and the horizontal slope. These considerations enclose the two main objectives of this paper. First, the definition of an algorithm to automatically detect layover zones, in order to identify low accuracy portions of an urban interferometric DEM. Second, the exploitation on a building-by-building base, in order to interpret the signal return and provide layover decomposition.

\section{INSAR MODEL}

The simplest building shape is a rectangular cuboid, with the ground, roof, and wall represented by the lower and higher horizontal and the vertical segments, respectively, as in Fig. 2. In the layover area, the signal return is a superposition of these contributions. In the interferometric framework, for the master satellite, the slant range distance $R_{0}$ between the satellite and the three layover scatterers is not varying by definition. On the contrary, three different distances are measured between slave satellite and points on ground $\left(R_{1}\right)$, wall $\left(R_{2}\right)$, and roof $\left(R_{3}\right)$. 


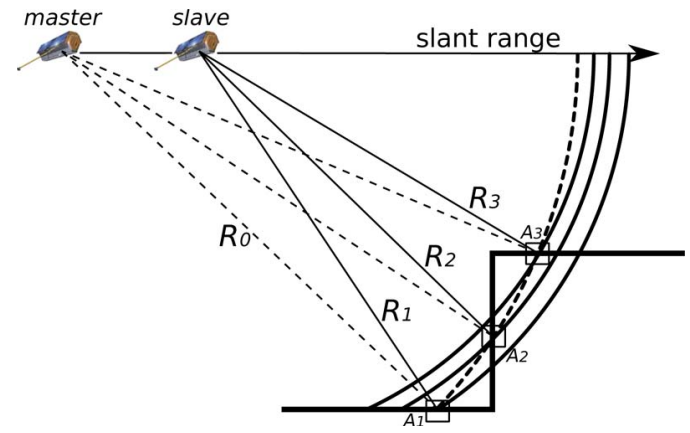

Fig. 2. Interferometric signal model for a building layover pixel. $R_{1}$ is the slant range distance between the satellite and the ground scatterer $A_{1}$, similarly for the wall scatterer $\left(R_{2}, A_{2}\right)$ and the roof scatterer $\left(R_{3}, A_{3}\right)$. $R_{0}$ is the master distance between the satellite and the three scatterers.

Thus, master $s_{m}$ and slave $s_{s}$ focused signal at the range $r$ and azimuth $x$ in the layover area are [22]

$$
\begin{aligned}
s_{m}(r, x) & =\sum_{i=1}^{3} A_{i}(r, x) \exp \left\{-j 2 k R_{0}(r, x)\right\} \\
s_{s}(r, x) & =\sum_{i=1}^{3} A_{i}(r, x) \exp \left\{-j 2 k R_{i}(r, x)\right\}
\end{aligned}
$$

where $k$ is the wavenumber, and $A_{i}$ is a complex variable, including the bidimensional system impulse response and the local backscatter, which are assumed equal in both geometries. ${ }^{1}$

The interferometric phase is the argument of a sum of nine complex terms

$$
\begin{aligned}
& \phi(r, x)= \arg \left(s_{m}(r, x) s_{s}^{*}(r, x)\right) \\
&=\arg \left(\sum _ { i = 1 } ^ { 3 } \left[\left(A_{i}^{*} \sum_{j=1}^{3} A_{j}\right)\right.\right. \\
&\left.\left.\quad \cdot \exp \left\{-j 2 k\left(R_{i}(r, x)-R_{0}(r, x)\right)\right\}\right]\right) .
\end{aligned}
$$

The analytic derivation of (2) without further approximations is not bringing to a compact expression. Instead, simulations and test on real data have been conducted [24]. Equation (2) is plotted in Fig. 3 assuming $A_{i}$ as a real constant for simplicity. The factors $A_{i}$ are considered as weights to the exponential terms, i.e., they represent the impact of the single layover components in the signal. The interferometric phase is a decreasing function for increasing slant ranges in case of positive height of ambiguity. A noticeable phase gradient is at the layover beginning, with a singular exception for total ground dominance $\left(A_{2}=A_{3}=0\right)$. Total wall dominance $\left(A_{1}=A_{3}=0\right)$ yields the highest gradient. Mixed weights produce phase jumps with spreads depending on the actual backscattering configuration. The absolute phase layover trend is nonlinear, with the exception of single component dominance.

\footnotetext{
${ }^{1}$ Backscattering variations are measured in case of different ambient conditions, different system parameters or target changes. For small baselines, in an urban scenario, slight changes may be expected for the ground contribution, particularly in a dual-pass interferometric configuration. In a single-pass configuration, very little changes or no changes are expected [23].
}

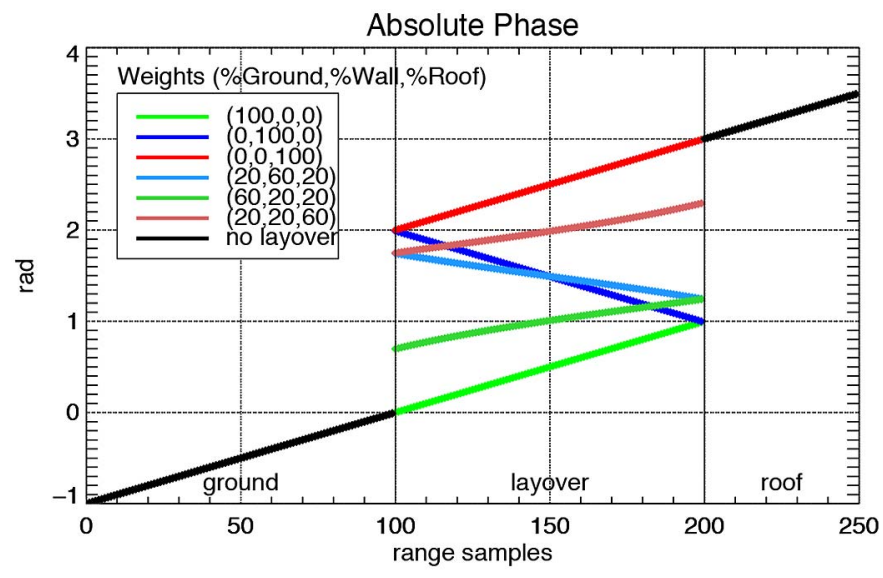

Fig. 3. Noise-free absolute phase simulation of a step function considering various exponential weights for the different segments composing the function. Ground, wall and roof represent the lower horizontal, the vertical and the higher horizontal segments, respectively. In the layover area, six cases are considered with different colors.

The range spectrum of the interferogram $I\left(f_{r}\right)$ can be derived by Fourier-transforming (2) in the range dimension

$$
\begin{aligned}
I\left(f_{r}\right)= & \sum_{i=1}^{3}\left[S_{m}\left(f_{r}\right) * S_{s}^{*}\left(-f_{r}\right)\right] \\
\simeq \sum_{i=1}^{3}[ & R_{i i}\left(f_{r}-\Delta f_{r_{i}}\right)+C_{\mathrm{in}}\left(f_{r}-\Delta f_{r_{i}}\right) \\
& \left.+C_{\mathrm{im}}\left(f_{r}-\Delta f_{r_{i}}\right)\right], \quad\left\{\begin{array}{l}
n \neq m \neq i \\
(n, m) \in[1,2,3]
\end{array}\right.
\end{aligned}
$$

where $R_{i i}\left(f_{r}\right)$ is the autocorrelation of the $i$ th layover scatterer spectrum and $C_{\mathrm{in}}\left(f_{r}\right)$ and $C_{\mathrm{im}}\left(f_{r}\right)$ are the cross correlations between the $i$ th and the $n$th and the $m$ th spectra, respectively. The spectra are bandpass filtered by the end-to-end SAR transfer function, approximated by a rectangular window [22], and shifted by $\Delta f_{r}$. System noise has not been considered in (1)-(3). Considering Gaussian noise, its impact is to add to (3) a broad triangular pattern centered on zero. $\Delta f_{r}$ is proportional to the terrain slope $\alpha$, according to [25]

$$
\Delta f_{r_{i}}=-\frac{f_{0} \Delta \theta}{\tan \left(\theta-\alpha_{i}\right)}=-\frac{f_{0} \Delta \theta}{\tan \left(\theta_{\text {inc }_{i}}\right)}
$$

where $\Delta \theta=B_{n} / R_{0}$, being $B_{n}$ the normal baseline between the satellites, $f_{0}$ the carrier frequency, $\theta$ the master looking angle and $\theta_{\text {inc }}$ the local incidence angle. Equation (4) is valid also for the high-resolution urban scenario, although in principle, it was derived for distributed scattering, and it is currently the base of the spectral shift filtering operation in every InSAR processor [26]. High-resolution urban scenes are characterized by a set of coherent scatterers lying on planar surfaces, e.g., on a facade. These scatterers, having a stable phase, geometrically provide a set of differential paths, which is proportional to the slope, in which the scatterers are located, according to (4). An explicit example is in given Fig. 1(b), where the sequence of skyscraper window eaves at the vertical slope yields an interferometric phase with a clear fringe frequency. The same consideration 
can be addressed for roof scatterers. Their radiometric pattern, hence their impact in the interferogram spectrum in (3), depends on their physical structure and cannot be uniquely defined. For instance, ground scattering may be locally a distributed one.

Thus, for the simple building model in Fig. 2, the frequency corresponding to the ground $\Delta f_{\text {ground }}$ is equal to the roof one $\Delta f_{\text {roof }}$ since they both have zero slope. A relationship between the flat ground and wall frequencies can be established considering the $90^{\circ}$ separation

$$
\theta_{\text {inc }}^{\text {ground }}=-\left(\frac{\pi}{2}-\theta_{\text {inc }}^{\text {wall }}\right) \text {. }
$$

In the frequency domain, (5) corresponds to a negative frequency for the ground/roof and a positive one for the wall (in the sign convention of (4)). Consequently

$$
\begin{aligned}
\Delta f_{\text {ground }} & =-\frac{f_{0} \Delta \theta}{\tan \left(\theta_{\text {inc }}\right)} \\
\Delta f_{\text {wall }} & =-\frac{f_{0} \Delta \theta}{\tan \left(\theta_{\text {inc }}-\frac{\pi}{2}\right)}=f_{0} \Delta \theta \tan \left(\theta_{\text {inc }}\right) \\
& =-\Delta f_{\text {ground }} \tan ^{2}\left(\theta_{\text {inc }}\right) .
\end{aligned}
$$

Equation (6) reveals that for this kind of analysis, it is important not to demodulate the interferogram for the flat Earth component, i.e., for $\Delta f_{\text {ground }}$. The estimation of the frequencies and the corresponding terrain slopes is described in Section V.

\section{LAYOVER GEOCODING}

The geocoding stage is usually the last stage of an InSAR processor. The absolute phase is converted here in surface elevation and georeferenced on a specific datum. Several methods were proposed (e.g., [27] and [28]). The geometric principle of this operation is simple. A SAR sensor images a point on Earth at a particular range. All the points located on a circle with a ray centered at the sensor position are imaged at the same range, thus yielding infinite solutions. In an InSAR framework, this ambiguity is solved by imaging the same point with a second sensor. The unwrapped InSAR phase defines hyperbolas having equal range differences between the sensors. Consequently, on the Doppler plane, a point on Earth is located at the intersection between an iso-range-difference hyperbola and a iso-range circle. This relationship yields the generation of a digital interferometric elevation model. An example of flat-Earth mapping is shown in Fig. 4(a).

This basic geometrical relationship can be also exploited for the layover case. As shown in Fig. 3, building layovers generally generate high absolute phase gradients at their beginning and their end. In Fig. 4(b), the focus is on the mapping of such a trend, in case of total vertical dominance. In this circumstance, the mapping of the SAR domain in the cartographic one is sparse, i.e., with a variable distance between mapped points. As effect, a DEM derived with a bilinear interpolation between points presents artificial ramps. In this example, the derived DEM points are shown in blue. The generated elevation points from the absolute phase are in red. The height of the elevation points, and consequentially the artificial model slopes, depends on the layover contributors weighting in the returned signal. A

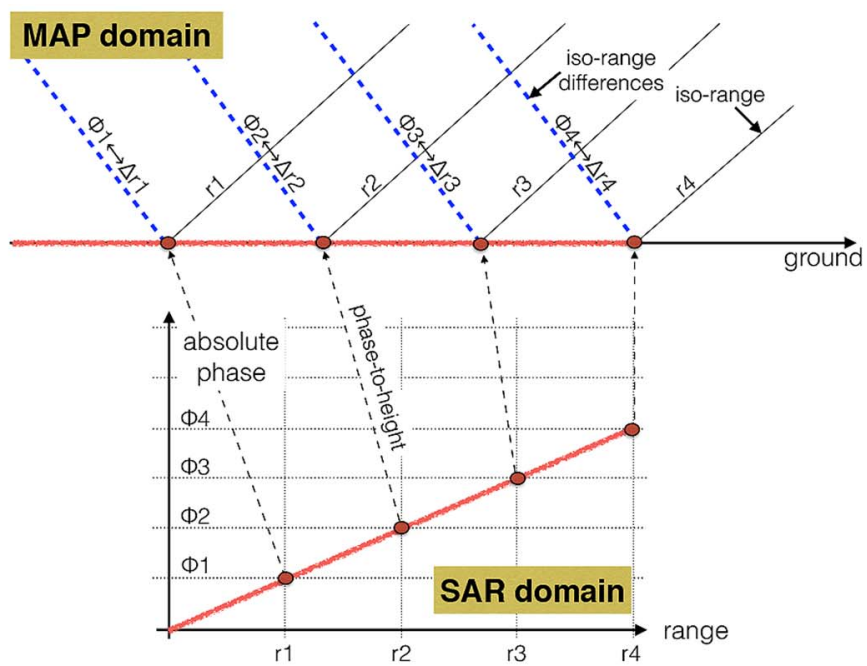

(a) flat earth mapping

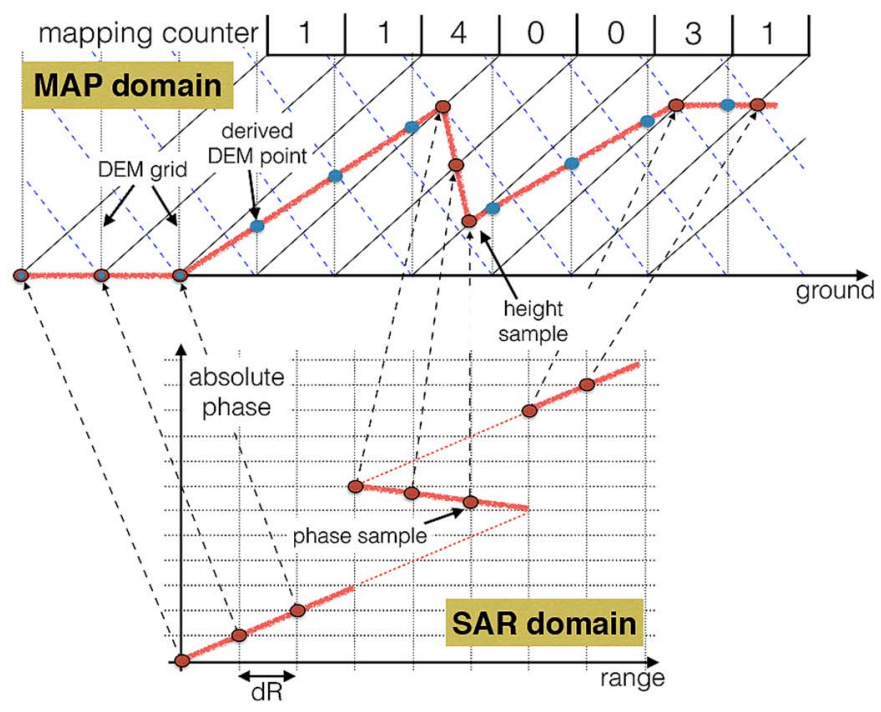

(b) layover mapping

Fig. 4. SAR absolute phase mapping in a geographic domain. (a) Flat Earth mapping. Geographic points are determined at the intersection between isorange circles and iso-range-differences hyperbolas, here drawn as straight lines. (b) Layover mapping. A phase gradient yields an elevation model ramp when employing a bilinear interpolation for the derivation of DEM points.

real example of interferometric urban elevation model with the highlighted effect is in Fig. 5(a). All the buildings imaged in this portion present the layover artifact, as highlighted for one of them with a red circle. A range section of the corresponding absolute phase is in Fig. 5(b). The real trend fits well with the simulated one in Fig. 3. A detailed study on the model accuracy is in [12]. Evidently, accuracy is limited at layover locations.

\section{LAYOVER DETECTION}

\section{A. Algorithm}

The derivation of the layover portion directly from the interferometric phase, e.g., searching for a high-phase gradient and subsequent phase slope, may be difficult due to phase noise superimposed to signal. The proposed procedure exploits 


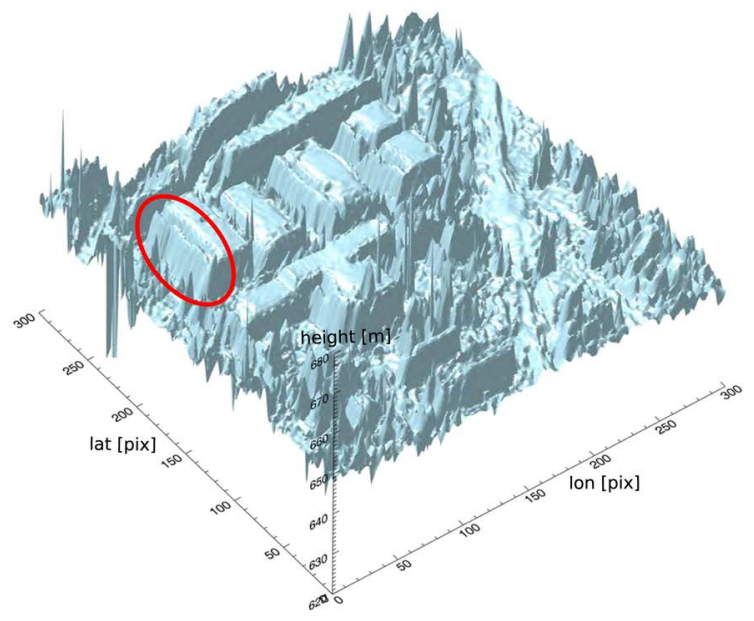

(a)

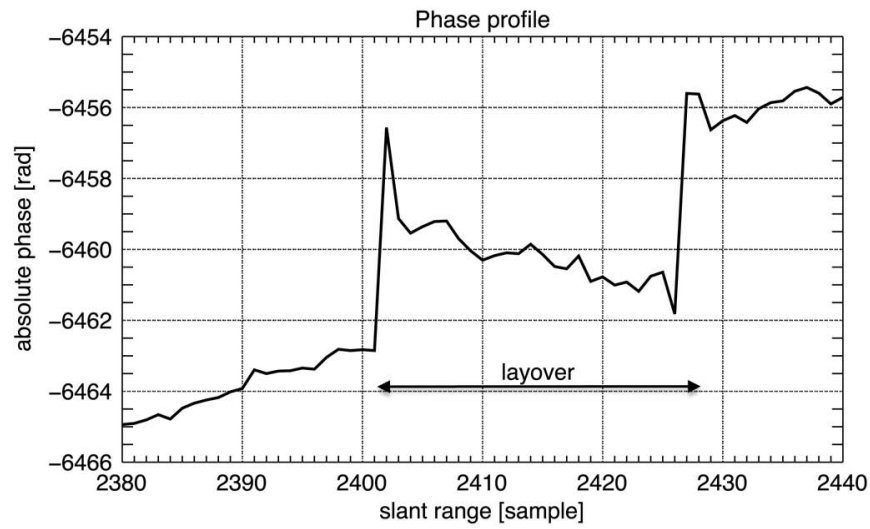

(b)

Fig. 5. (a) Three-dimensional interferometric elevation model portion over Las Vegas generated with spotlight TanDEM-X data. Layover ramps are marked for a building with a red circle. (b) Range section of the InSAR absolute phase for the highlighted building. (a) Elevation model. (b) absolute phase.

instead the geocoding algorithm described in the previous section and, in particular, a subproduct generated for the purpose and named mapping counter. The mapping counter is a map, in slant range coordinates, whose samples $m_{c}$ describe the number of occurrences of a SAR interferogram pixel in the produced DEM. For a flat terrain, the SAR mapping on the DEM raster depends on the DEM posting and the subsampling used in the interferometric processing. In particular, the number of SAR pixels contributing to a DEM cell is

$$
n_{\mathrm{SAR}}=\frac{1}{2}\left(\frac{\Delta_{\mathrm{DEM}}^{\mathrm{LON}}}{\Delta_{\mathrm{InSAR}}^{g r_{r g}}} \frac{\Delta_{\mathrm{DEM}}^{\mathrm{LAT}}}{\Delta_{\mathrm{InSAR}}^{a z}}\right)
$$

where $\Delta_{\mathrm{DEM}}^{\mathrm{LON}}$ and $\Delta_{\mathrm{DEM}}^{\mathrm{LAT}}$ represent the DEM posting for the northing and easting direction, and $\Delta_{\mathrm{InSAR}}^{g r_{r g}}$ and $\Delta_{\mathrm{InSAR}}^{a z}$ the interferogram sampling in ground-range and azimuth dimensions, respectively. For an ideally flat terrain and noiseless interferogram, in case of $n_{\mathrm{SAR}}=1$, every SAR pixel is used just once, and the mapping counter is a unit matrix. A divergence with this condition is an indicator of slopes. In the general case, the interferometric DEM exhibits slopes when the mapping counter pixel $m_{c}$ differs from the integer part of $n_{\mathrm{SAR}}$. Any terrain slope, also not in layover, impacts in the homogeneity of

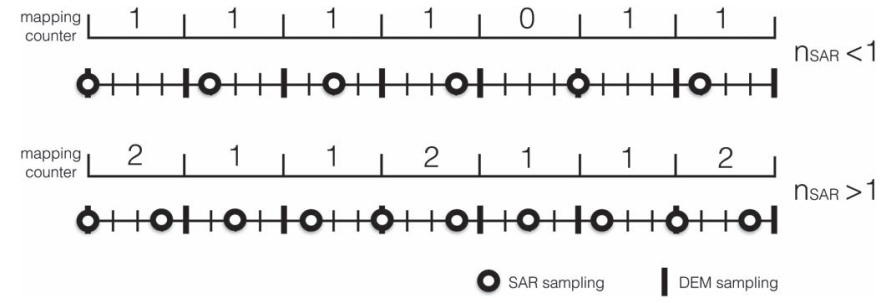

Fig. 6. Mapping counter measured in presence of a nonzero fractional part $\left\langle n_{\mathrm{SAR}}\right\rangle$ and an integer part equal to 1 . At the top, $n_{\mathrm{SAR}}<1$ yields a regularity of no-mapping pixels. At the bottom, $n_{\mathrm{SAR}}>1$ yields a regularity of multiplemapping pixels. The SAR sampling in the DEM raster is showed below the mapping counter.

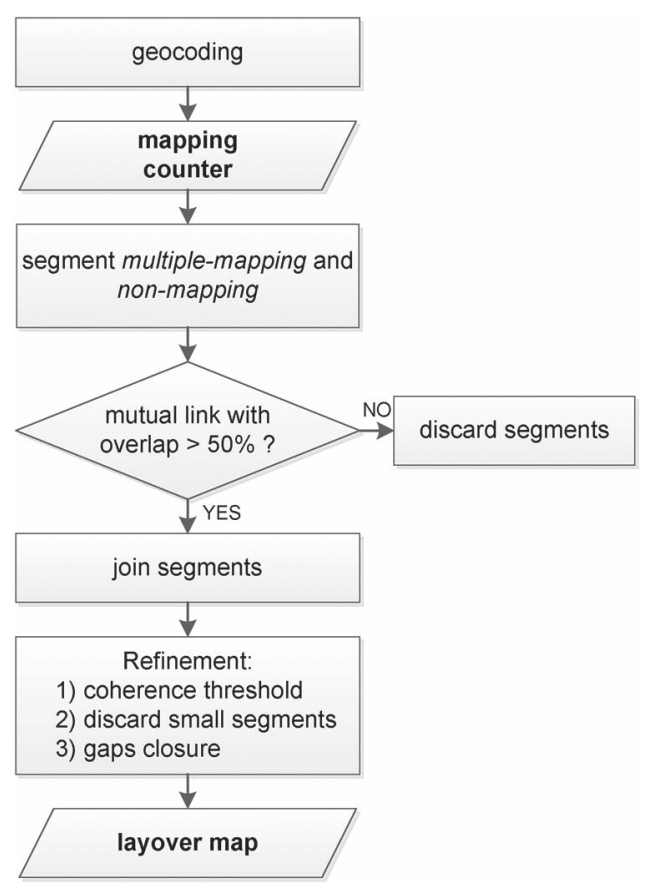

Fig. 7. Block scheme for the proposed building layover detector.

the mapping counter. Consequently, the detection of building layovers by inspecting the mapping counter results is more accurate in case of flat or locally flat terrains. To be remarked, the counter records integer values whereas $n_{\mathrm{SAR}}$ has usually a nonzero fractional part $\left\langle n_{\mathrm{SAR}}\right\rangle$. Thus, even for flat terrain, a discrepancy is recorded with a pattern depending on the ratio between the SAR and the DEM grids. Fig. 6 depicts the discrepancy measured in the mapping counter considering a nonzero fractional part and an integer part equal to 1 . In the figure, the fractional part is negative and positive for the upper and lower example, respectively. It is then relevant, for the effectiveness of the algorithm, to set the interferometric processor in order to have $\left\langle n_{\mathrm{SAR}}\right\rangle$ close to zero.

The block scheme of the proposed algorithm is in Fig. 7. The mapping counter shown at the top of Fig. 4 (b) (here $n_{\mathrm{SAR}}=1$ ) can be taken as reference. A high-phase gradient creates a multiple-mapping region, i.e., $m_{c}>n_{\mathrm{SAR}}$. A straightforward technique to extract layovers is then used to detect pixels accomplishing this condition for every slant range line of the mapping counter. This constraint identifies the beginning of 
a slope, or, in our case, a building layover zone. The following mapping counter pixels shall accomplish the condition $m_{c}<n_{\mathrm{SAR}}$. The detection implies a segmentation of the two regions and a mutual-link search. Flexibility in the mutual link must be introduced as phase noise, or a particular contributor weighting set, may disjoint them. Thus, a minimum overlap is set to $50 \%$ to increase the detection rate. This flexibility experimentally doubled layover detections (see next section). A set of refinements finalizes the layover map. If the building is large, then the nonmapping region must be enclosed in between two multiple-mapping regions by cause of the phase gradient at the end of the layover zone (see Fig. 3). In contrary, if the building is totally under the layover effect, then also its shadow area can accomplish the condition $m_{c}<n_{\mathrm{SAR}}$, thus falsely enlarging the estimated layover patch. In the latter case, the interferometric coherence is exploited in order to define the layover ending point through a threshold, approximating the coherence estimation bias value when the true coherence is zero [29]

$$
t_{0}=\frac{1}{2} \sqrt{\frac{\pi}{N_{c}}}
$$

where $N_{c}$ is the number of resolution cells used to estimate the coherence. Pixels having coherence lower than $t_{0}$ are considered as shadow pixels. Noisy areas may generate high-phase gradients and consequently wrong detections in the mapping counter. An efficient phase reduction algorithm (e.g., an adaptive multilooking technique) should be used to attenuate artifacts. Nevertheless, small false detections, as isolated layover coming from trees adjacent to buildings, can always be discarded considering the minimum spatial support of a standard building in the SAR interferogram. Finally, every detected building layover patch is made consistent by closing remaining gaps.

\section{B. Example}

The layover detection algorithm is tested for an interferometric TanDEM-X scenario. A bistatic spotlight acquisition taken on the January 4, 2012 over the city of Berlin (Germany) is chosen. The satellites had a normal baseline of about $110 \mathrm{~m}$ yielding a height of ambiguity of $65 \mathrm{~m}$. The geometrical and processing parameters are in Table I. The same data set was used in [12] to test the TanDEM-X DEM generation capabilities over urban areas. As this paper's purpose is to work on a building-by-building base, the spotlight mode is of fundamental importance due to the high-resolution capable to isolate building signatures. Moreover, the bistatic configuration is, as well, favorable to circumvent false detection resulting from temporal decorrelation.

The detection of layover zones starts with the generation of the mapping counter. For a correct analysis of the map, the number of SAR pixels in the interferogram contributing to a DEM cell must be computed. The ground range and azimuth interferogram sampling are $\Delta_{\text {InSAR }}^{g r_{r g}}=2.03 \mathrm{~m}$ and $\Delta_{\text {InSAR }}^{a z}=$ $2.60 \mathrm{~m}$, respectively. The TanDEM-X processing [30] is set to generate a DEM with longitude and latitude postings of $\Delta_{\mathrm{DEM}}^{\mathrm{LON}}=2.16 \mathrm{~m}$ and $\Delta_{\mathrm{DEM}}^{\mathrm{LAT}}=2.37 \mathrm{~m}$. According to $(7), n_{\mathrm{SAR}}$
TABLE I

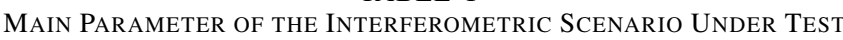

\begin{tabular}{rl}
\hline parameter & value \\
\hline PRF & $8200 \mathrm{~Hz}$ \\
range bandwidth $f_{s}$ & $300 \mathrm{Mhz}$ \\
center incidence angle (master) & $41.8 \mathrm{deg}$ \\
height of ambiguity & $+65.4 \mathrm{~m}$ \\
data pixel spacing (range, azimuth) & $0.45,0.86 \mathrm{~m}$ \\
multi-look algorithm & IDAN \\
total number of looks & 20 \\
interferogram resolution & $2.72 \mathrm{~m}$ \\
mean coherence & 0.69 \\
ground frequency $\Delta f_{\text {ground }}$ & $5.26 \mathrm{MHz}$ \\
wall frequency $\Delta f_{\text {wall }}$ & $-4.14 \mathrm{MHz}$ \\
\hline &
\end{tabular}

results 0.9877. Thus, following the guidelines in Section III, building layover is defined for mapping counter range segments composed of samples with values larger than one (tagged as multiple mapping). In Fig. 8 the mapping counter of the full acquisition is shown at the top left. In this case, no filtering is applied since the interferometric phase is generated using an adaptive-multilooking algorithm (IDAN) to strongly reduce speckle [31]. The map is quantized on three levels: a null value, in black, a unit value, in gray, and higher values in white. These last values, representing multiple mappings, are extracted and shown in the center-left map. In addition to this paper purposes, they can be used to estimate the building orientation in case of rectangular shapes. A regularity of diagonal multiple mappings is visible. This is the consequence of a nonzero fractional part $\left\langle n_{\mathrm{SAR}}\right\rangle$ and will be automatically discarded in the layover map refinement. At the bottom left, the $m_{c}<n_{\mathrm{SAR}}$ patches (tagged as nonmapping as $m_{c}=0$ ) are extracted and coded in white. Mainly representing building shapes, they contain the larger part of the desired information. The refinement described in the previous section yields a detection of single building layovers. The result is the layover map shown at the bottom right. No a priori assumptions have been made about building shapes. The SAR amplitude of the master channel is also shown on top right to highlight the difficulties encountered by algorithms based only on simple amplitude and coherence thresholds (e.g., the layover portion of a building may be smaller than the full building patch). As a final remark, the additional processing time required for the generation of the layover map in the interferometric chain (from focused data to DEM) is negligible. This algorithm can be then easily integrated in an InSAR processor straight after the geocoding stage.

\section{Fringe-Frequency Estimation}

Once building layovers are extracted, they can be further analyzed by inspecting their spectral properties, in order to find the physical slopes superimposed in every detected patch. The frequencies characterizing the building layover spectrum in (3) can be estimated and the related ground slopes can be then derived by inverting (4)

$$
\alpha=\arctan \left(\frac{f_{r g} \tan \theta+f_{0} \Delta \theta}{f_{r g}-f_{0} \Delta \theta \tan \theta}\right) .
$$



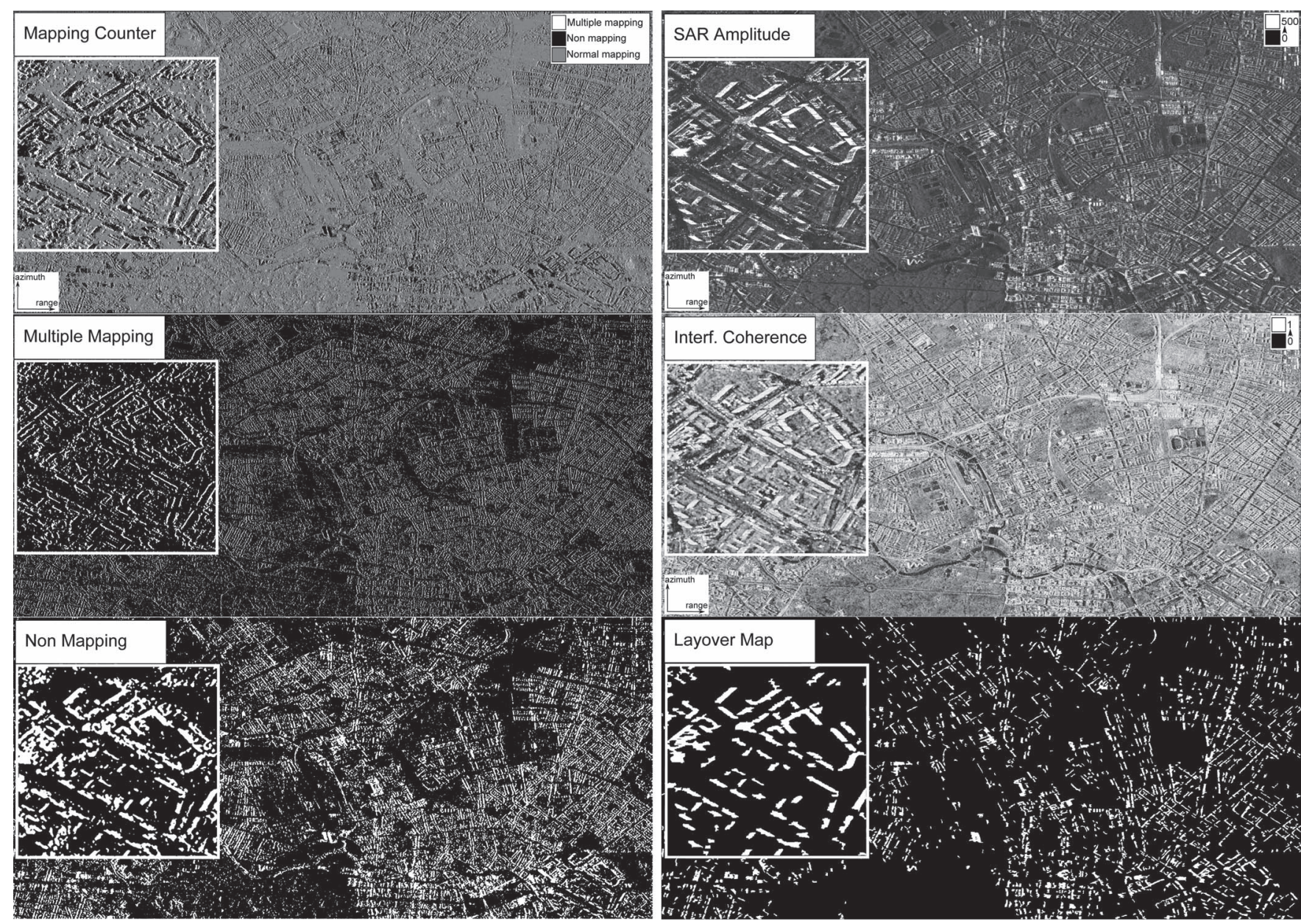

Fig. 8. Visual representation of the layover detection processing steps. (Top left) Mapping counter. $n_{\mathrm{SAR}}=0.99$. In black the nonmapping areas having value $m_{c}=0$, in gray the normal mapping areas having value $m_{c}=1$ and in white the multiple mapping areas having value $m_{c}>1$ are represented. (Middle left) Multiple mapping areas extracted from the mapping counter are in white. (Bottom left) Nonmapping area extracted from the mapping counter are in white. (Top right) SAR amplitude of the master channel in SAR coordinates. (Middle right) Interferometric coherence. (Bottom right) Final building layover map. The detected and segmented buildings are white coded. For all the figures the highlighted portion is a zoom of the south-east part of the map.

The number of layover contributors per building is also assessed during the estimation, assuming the building composed by planar facets. Ideally, approximating (2) at the first order, i.e., $\phi(r, x) \simeq \phi_{r}^{\prime} d r+\phi_{x}^{\prime} d x$, and solving for the local backscatter, layover heights can be determined by making use of the SAR phase-to-height conversion [22]. In the following, it is shown that the complete layover decomposition is impracticable with a sole interferogram because of the very small range layover support. Nevertheless, the dominant signal in the building layover can be extracted with a certain accuracy depending on the estimation technique, the number of layover range and azimuth pixels and the local SNR ratio.

\section{A. Simulations}

Two classical methods are analyzed in this paper: fast Fourier transform (FFT) (or periodogram analysis) and conventional MUSIC. The latter is a parametric method that fits with the interferogram model in (2). As both of the algorithms are very common and widely used in the spectral estimation framework, they will not be described in this paper. Details on the methods can be found in several dedicated books, e.g., [32] and [33].
The estimation is performed for every detected building layover, in the slant range domain. Each slant range line is assumed as an independent realization of the same process. This assumption implies a building modeled by planar surfaces, the same for every range line. To be noticed, with this assumption building orientation does not impact the estimation. The sole repercussion lies in the estimation of nonvertical and nonhorizontal slopes (e.g., gabled roofs): the estimated roof slope is the slope projected in the range direction and not the real one.

A complexity comes from the varying range support. Indeed, the realization dimensions, i.e., the number of range samples for every azimuth line, may vary depending on the building structure. In contrast, a standard frequency estimation usually assumes a constant size for each realization and then averages over the estimates. This issue is circumvented by assigning a specific weight for every realization, depending on its size. Larger realizations have larger weights. A minimum size is also defined. Realizations smaller than the minimum size are discarded. In the FFT algorithm, the set of range spectra of a building patch is computed over a defined frequency support, so that a simple weighted average is straightforward. In the MUSIC algorithm, the correlation matrix is estimated with a spatial 


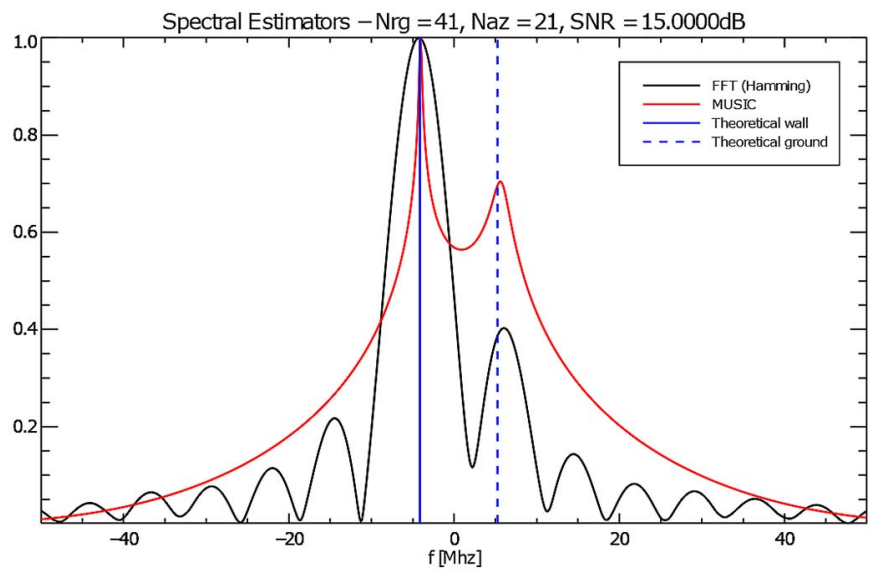

Fig. 9. Simulated spectrum (black) and pseudospectrum (red) for a bitonal signal. The simulated frequencies represent the wall frequency (blue) and ground frequency (dashed blue). The range spatial support is 41 samples. Spectra are averaged over an azimuth spatial support of 21 samples. SNR is set to $15 \mathrm{~dB}$.

smoothing method [34] in order to use all the samples of valid realizations. The realization weight is then set as proportional to the number of smoothings per building range line: the larger the realization, the larger the number of smoothings, the larger the weight.

The scope of the following analysis is to demonstrate the potentials and the limits of the two algorithms for the particular case of the cuboid building model. A primary objective is the definition of the minimum realization size $n_{\text {lay }}^{\min }$ for an accurate slope estimation for the TerraSAR-X/TanDEM-X highresolution spotlight case. For this purpose, a simulation of a sum of two sinusoids with frequencies $\Delta f_{\text {ground }}$ and $\Delta f_{\text {wall }}$ is performed. The model in (2) is approximated at the first order with a complex sum of two tones with frequencies $\Delta f_{\text {wall }}$ and $\Delta f_{\text {ground }}$ with real valued amplitudes. Amplitudes determine the backscatter of the wall and the ground/roof. SNR is defined by adding white Gaussian noise. Parameters of the simulations are given in Table I.

An unlimited number of different combinations can be simulated. A strong tone mixed up with a weak one is chosen as representing many cases analyzed in the next section. An exemplary simulation result is in Fig. 9. Here, one could visually evaluate the better MUSIC performance when compared with FFT in case of dominance of one frequency (in this case the wall one), providing two clear peaks at the frequency locations. Theoretically, considering the FFT resolution, the periodogram detection of two tones with equal weights requires a number of samples larger than

$$
n_{\text {lay }}^{\min }>\frac{f_{s}}{\left|\Delta f_{\text {ground }}-\Delta f_{\text {wall }}\right|}
$$

equal to 31 samples in our test case. A simulation is performed to evaluate the minimum range support at four different SNR levels $(0,5,10,15 \mathrm{~dB})$ for the two algorithms. In Fig. 10 the estimated absolute errors $\mid \widehat{\Delta f}$ wall $-\Delta f_{\text {wall }} \mid$ and $\left|\widehat{\Delta f}_{\text {ground }}-\Delta f_{\text {ground }}\right|$ are plotted for the four cases using a fixed azimuth support of 21 samples and 1000 simulation
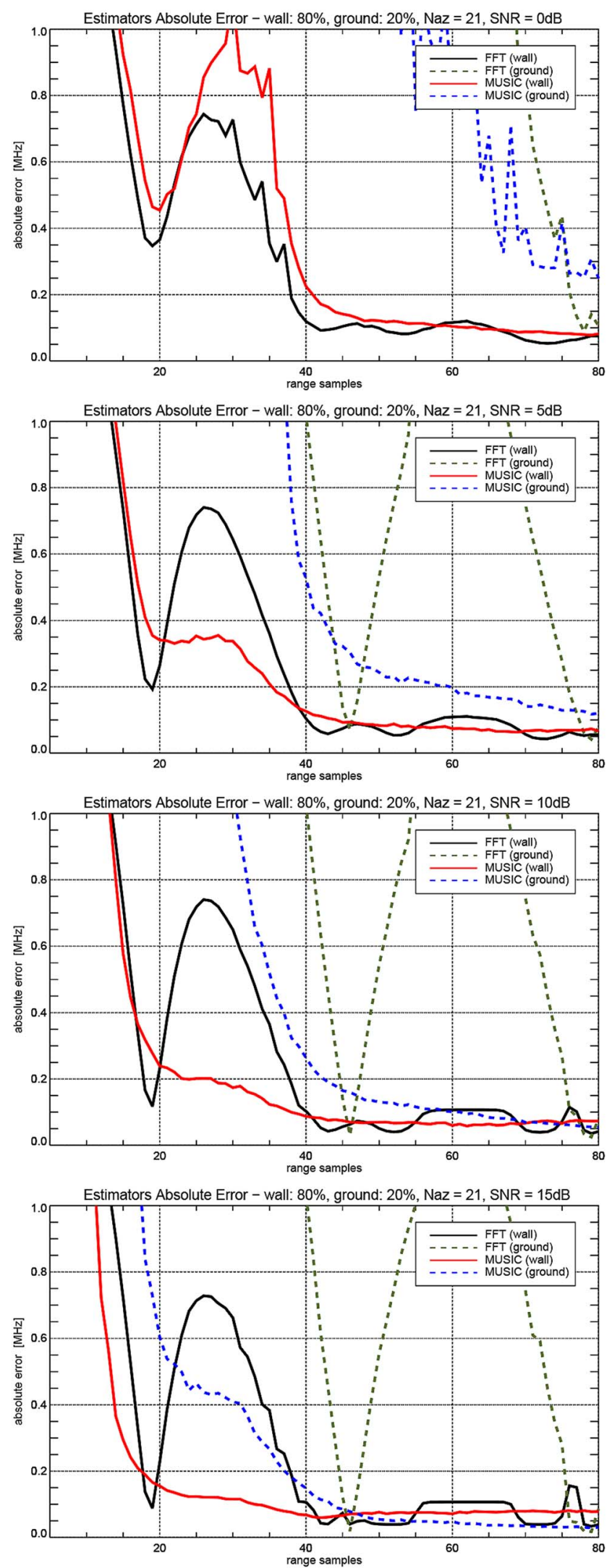

Fig. 10. Fringe-frequency absolute error for varying range support for FFT and MUSIC algorithms. Wall frequency is dominant in the simulation with a $80 \%$ weight (black and blue lines for FFT and MUSIC, respectively). Ground frequency has a $20 \%$ weight (dashed green and blue lines for FFT and MUSIC, respectively). SNR is set to $0,5,10$, and $15 \mathrm{~dB}$ (top to bottom). 21 azimuth samples are considered in averaging the estimates. 1000 simulations per range sample are performed. 
TABLE II

MiNimum RANGE SUPPORT $n_{\text {lay }}^{\min }$ FOR A $80 \%$ WALL WEIGHT AND A $20 \%$ GROUND WEIGHT

\begin{tabular}{|c|c|c|}
\hline & wall $^{a}$ & ground/roof $^{\mathrm{a}}$ \\
\hline$\left.\overline{\text { MUSIC }}\right|_{S N R=15 d B}$ & $12 / 14 / 17$ & $18 / 32 / 37$ \\
\hline MUSIC $\left.\right|_{S N R=10 d B}$ & $14 / 17 / 23$ & $32 / 37 / 43$ \\
\hline$\left.\operatorname{MUSIC}\right|_{S N R=5 d B}$ & $15 / 18 / 35$ & $38 / 42 / 60$ \\
\hline$\left.\operatorname{MUSIC}\right|_{S N R=0 d B}$ & $35 \mathrm{~b} / 37 / 41$ & $62 \mathrm{~b} / 75^{\mathrm{b}} /-$ \\
\hline
\end{tabular}

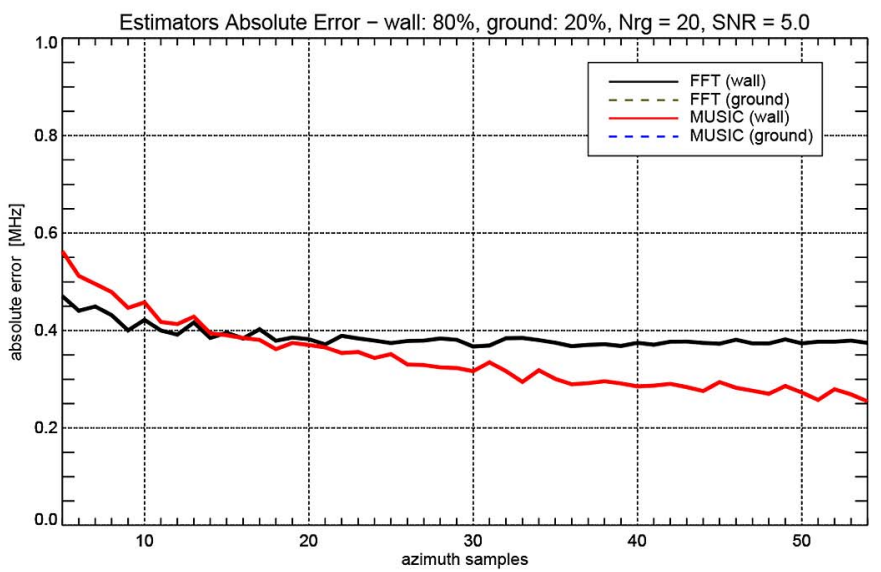

(a) azimuth support

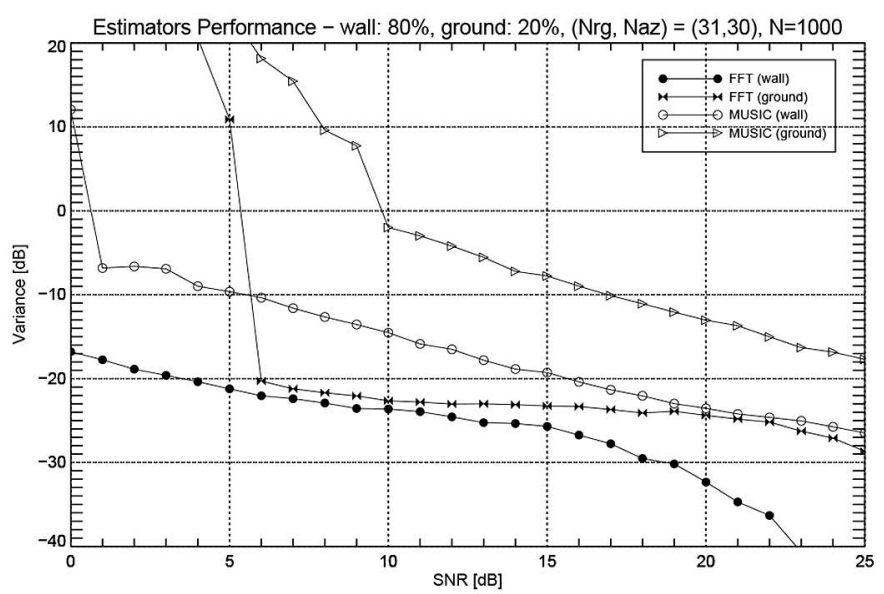

(b) estimator stability

Fig. 11. (a) Fringe-frequency absolute error for varying azimuth support for FFT and MUSIC algorithms. The range support is fixed to 20 samples. Wall frequency is dominant in the simulation with an $80 \%$ weight (black and blue lines for FFT and MUSIC, respectively). Ground frequency (20\% weight) is out of the plot. SNR is $5 \mathrm{~dB} .1000$ simulations per azimuth sample are performed. (b) Estimators stability-variance of the absolute error-at varying SNR for FFT and MUSIC algorithms.

runs for each range sample under test. It is not surprising to see how MUSIC outperforms FFT in terms of accuracy, particularly for the low weighted frequency. Generally, MUSIC has a strong dependence on SNR: for very low SNR values, FFT tends to outperform MUSIC. Nevertheless, the general superior performance in the detection recommends the use of MUSIC. In Table II, the MUSIC minimum support for three different accuracy levels is summarized.

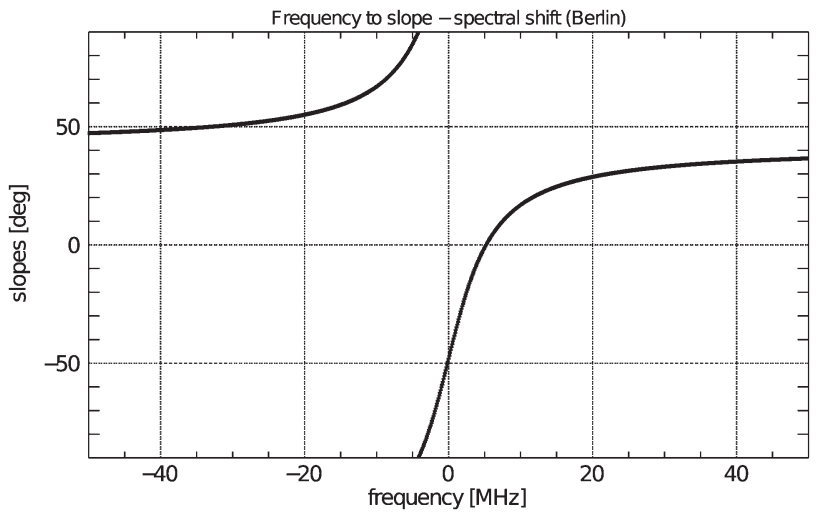

Fig. 12. Nonlinear relationship between ground slope and frequencies considering the data set used for the validation.

The azimuth support is used to average the frequency estimates. The impact in the absolute error is not as dramatic as the range support: in Fig. 11(a) a simulation at $5 \mathrm{~dB}$ reveals a decay of $0.2 \mathrm{MHz}$ for 20 azimuth samples for MUSIC, whereas the impact in the FFT is not relevant. Finally, a study on the estimator's stability at varying SNRs confirms the consideration made above: MUSIC is less stable at low SNRs. For both of the tones, FFT provides a smaller estimation variance [Fig. 11(b)].

\section{B. Principal Slope Estimation}

The previous analysis, summarized in Table II, remarked that frequency estimation of a multitonal signal requires quite a large spatial support, considering building layovers. Layovers extend for a number of pixels depending on the building height and the incidence angle. Smaller incidence angles and higher constructions provide larger supports. By definition, resolution increases the number of layover pixels. Additionally, the problem is also nonlinear, considering (4). In Fig. 12, the relationship between slopes and frequencies is depicted considering the system parameters in Table I. The estimation accuracy at different slopes varies significantly. For instance, a deviation of $0.4 \mathrm{MHz}$ in the frequency estimation for $90^{\circ}$ yields an error of about $2^{\circ}$, whereas the same inaccuracy for $35^{\circ}$ brings an estimation error of only $0.07^{\circ}$.

For all these reasons, proper layover decomposition and backscatter estimation is not feasible with a sole interferogram. Considering the simulations, the main layover component, when dominating over others, can be estimated with about 15 samples. Accurate secondary component estimation requires about a double number of samples, which makes a complete decomposition feasible only for high-rise and isolated buildings at X-band. Thus, only for the strongest component, i.e., the one with a higher backscatter, a single slope is estimated and denoted principal slope.

Since MUSIC provides frequency locations, but not an estimate of signal backscatter, a further estimation technique is required to detect the dominant frequency. First of all, the number of components is required as MUSIC input. For that, the Minimum Description Length (MDL) [32] has been chosen as selection technique. Considering the particular case, a maximum number of three components are considered. After that, if a single contributor is detected, then obviously, it is 


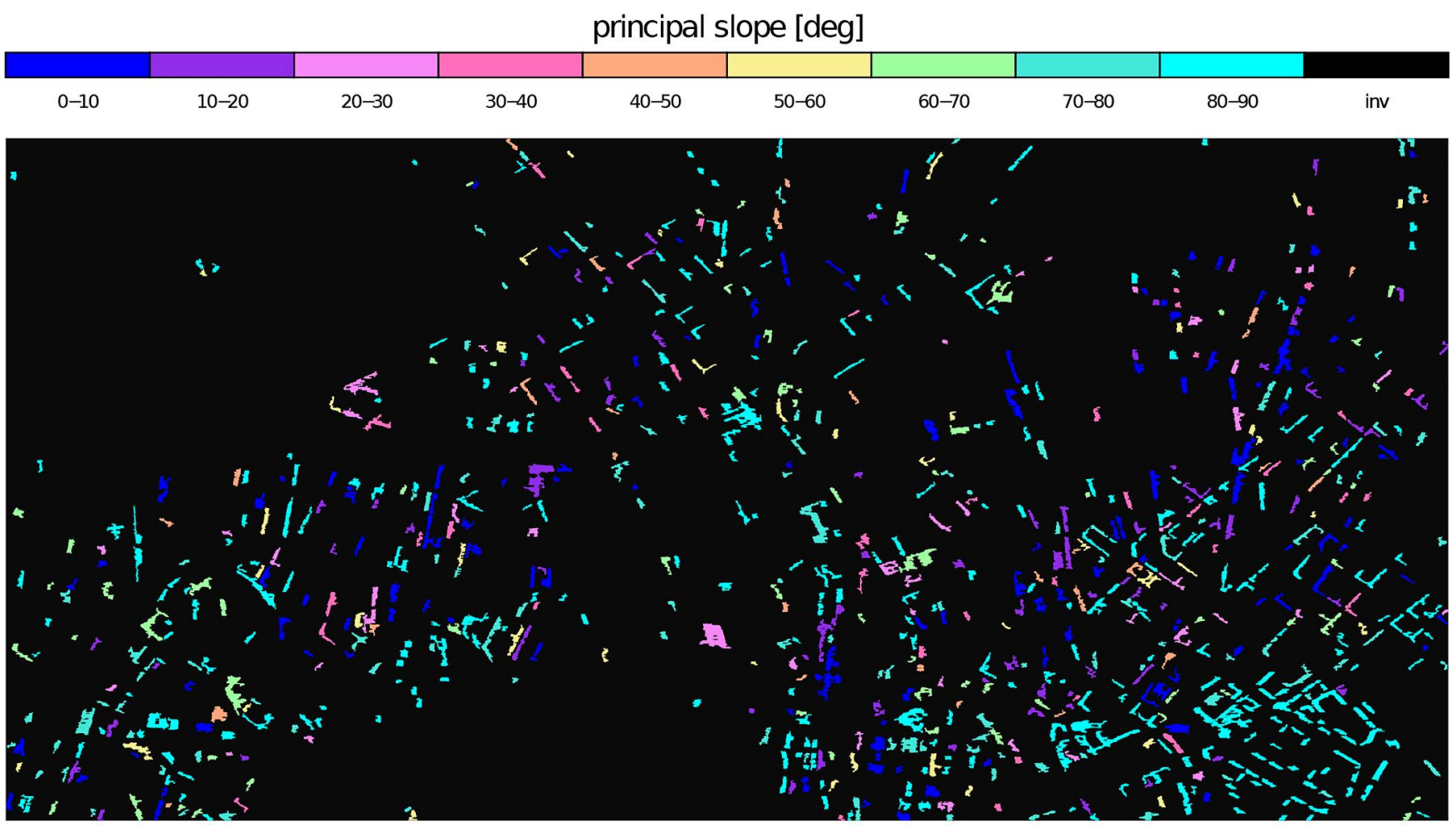

Fig. 13. Principal slope for the detected building layovers. A segmentation on the detected map and a conventional MUSIC algorithm is used to estimate the dominant frequency for layovers having a minimum range and azimuth support of 15 and 10 samples. Color scale is at the top.

the dominant one; otherwise, the dominant one is estimated by inverting the MUSIC model [33, p. 460].

\section{Example}

The frequency estimation framework is exploited to derive the principal slope of the layover portions. The test site is the same employed in Section IV-B. The principal slope, derived as in (9) by employing the conventional MUSIC algorithm, is shown in Fig. 13. Estimated slopes are regularly quantized in nine classes. Considering the performed simulations, the fringe frequency is estimated for layover patches having a minimum range and azimuth support of 15 and 10 samples, respectively. Discarded buildings can be visually recognized by comparing Fig. 13 and the bottom-right map in Fig. 8. The covariance matrix order is adaptively chosen depending on the actual range support. In particular, the order is defined as the minimum common support exceeding the minimum valid realization of 15 samples. The model order is estimated in the processing by adopting the MDL algorithm and fixing three tones as upper bound. In Fig. 14 the model order, i.e., the estimated number of layover components, is shown for the nine slope classes.

An analysis of the result indicates that for about $60 \%$ of the analyzed buildings (in total 866) wall has the dominance in the signal return. Flat roofs (or ground) dominate for about $25 \%$ and other slopes, as tilted roofs, for the remaining $15 \%$. A first consideration is about the generally larger wall support at the acquisition incidence angle (Table I) considering the buildings conformation of the city under analysis. In fact, excluding wall portions not visible by the radar due to local occlusion (e.g.,

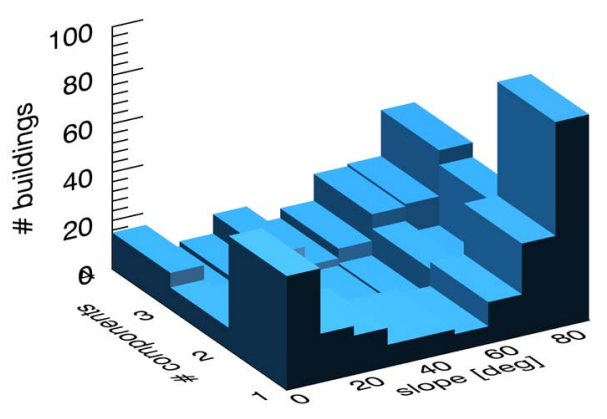

Fig. 14. Two-dimensional histogram of the estimated slopes and number of layover components. Axis represent the number of building layovers (z), the number of components $(y)$ and the estimated principal slope $(x)$.

trees or close buildings), the wall is generally totally included in the portion, whereas roofs are only summing up for a section of the total layover support. A second consideration is about the balconies-windows configuration, which creates a set of strong reflectors at the vertical slope. For these configurations, the facade layover contribution dominates over the others. An example is provided in Fig. 15. The mentioned buildings conformation is evident for this portion, representing the southerneastern part of the derived map and already taken as reference in Fig. 8. The derived spectrum and pseudospectrum for a benchmark structure are plotted at the bottom of the figure. A first-degree model is detected. The conventional MUSIC algorithm estimates a principal slope close to $90^{\circ}$. On the contrary, the FFT result is not accurately detecting the wall frequency. Generally, FFT results are much more sensible to local backscattering variations. 


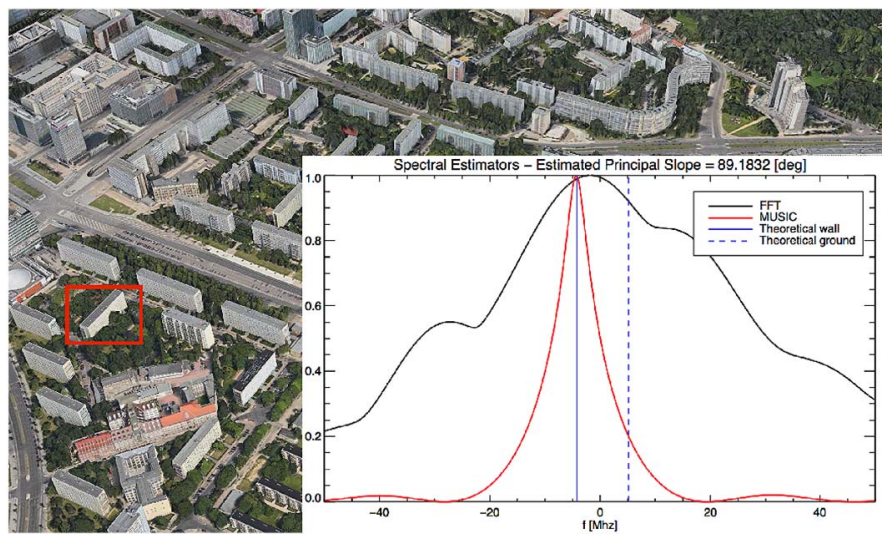

Fig. 15. Exemplary estimation of vertical slope dominance. The southerneastern part of the data set is shown with a 3-D optical view (Apple Inc.). The spectra of the building layover highlighted in red are overimposed. In red, the MUSIC pseudospectrum is used to obtain the principal slope estimation, very close to $90^{\circ}$. A single contributor is estimated. In black, the maximum of periodogram is also shown. Frequencies corresponding to the vertical and the horizontal slopes are represented with a continuous and a dashed blue line, respectively.

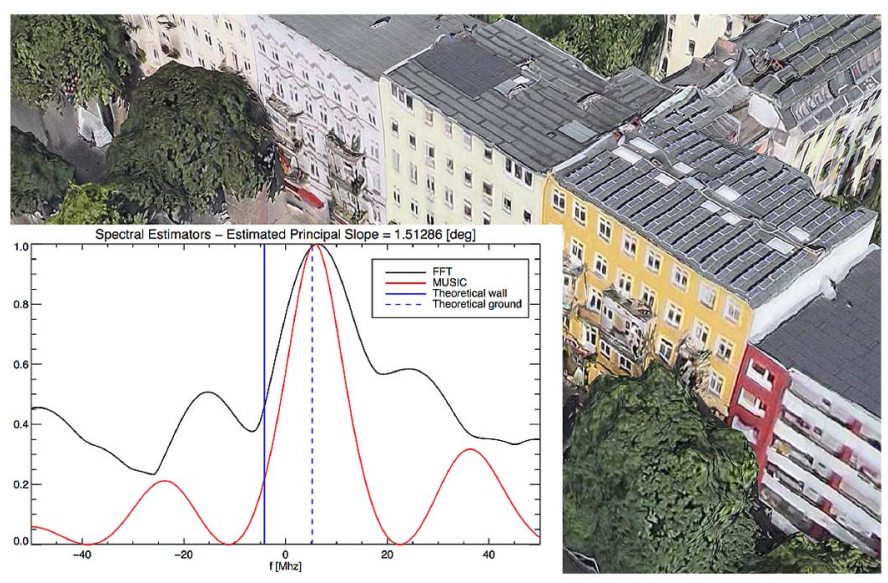

Fig. 16. Exemplary estimation of horizontal slope dominance. The buildings under analysis are shown with a 3-D optical view (Apple Inc.). The spectra of the yellow building are overimposed. In red, the MUSIC pseudospectrum is used to obtain the principal slope estimation, very close to $0^{\circ}$. Three layover contributors are estimated. In black, the maximum of periodogram is also shown. Frequencies corresponding to the vertical and the horizontal slopes are represented with a continuous and a dashed blue line, respectively.

Strong reflectors at the roof tops make the roof slope dominate. An example is in Fig. 16. The orange and the connected yellow building are belonging to a single layover patch. The adjacent red building is instead differently segmented due to a low coherence area that disjoins it. The horizontal slope is found as dominant, because of the set of solar panels and chimneys on the flat roof. The spectra here are more complex: in the layover patch also adjacent trees superimpose, and the vertical slope is not estimated. The other two slopes are not accurately estimated, once again demonstrating the inability to completely reconstruct the layover signal with a single interferogram. Nevertheless, the principal component provides useful information, e.g., the facet, in which strong scatterers are lying. This information is not easy to retrieve by inspecting the single amplitude, coherence and absolute phase. On the whole, it has been experimentally verified that when various scatterers at a specific facet exhibit a high backscattered signal return, their facet slope is measured and a single slope is estimated. Fig. 14 demonstrates that for the majority of the detected layovers a single contributor is estimated.

\section{CONCLUSION}

The mapping of urban areas with SAR sensors is of increasing attractiveness due to the increment of high-resolution data available. With new missions, as TanDEM-X, and expected future ones, urban SAR research is growing in a field dominated nowadays by optical or LiDAR sensors. In this context, this paper presents an algorithm for the detection of the layover portion of buildings. The focus is on the interferometric processor, and, in particular, on the geocoding stage. The particular layover absolute phase trend is exploited in the mapping matrices in a way to precisely identify layovers. The attractive points of the algorithm are the absence of a priori hypotheses and the lack of external high-resolution DEMs in input. These detections are also useful for an accuracy evaluation of InSARgenerated urban DEMs.

Spectral estimation is then considered in this paper as an additional instrument toward a better understanding of the physical phenomena behind the layover scattering decomposition. A super-resolution algorithm, MUSIC, is employed to derive the fringe frequencies characterizing the layover portion. Due to the limited estimation support, only the dominant frequency is found to be reliable information. The nonlinear relationship with slopes is employed to derive a principal slope map. A bistatic interferometric scenario is tested. It is found that for the layover's majority the facade contribution is the prevailing one due to the presence of targets with a high backscattered signal return at the vertical slope. Moreover, the number of layover contributors is assessed prior to the spectral estimation. It has been estimated that the signal return is dominated by a single contribution for the majority of the layovers.

The layover map application range is certainly wider than the one delineated in this paper. First, a change detection of the derived parameters (shape, slopes) for different temporal acquisition may support damage assessment applications. Second, geographical building orientation can be easily derived. Third, the result can be related to a persistent scatterer interferometry quality precheck. Fourth, the principal slope map and the estimation of the number of components can be an instrument for electromagnetic and simulation studies. Finally, as the scenario considered is the interferometric bistatic one, which main purpose is the generation of a DEM, the derived estimates can be used to improve the elevation model in the detected positions.

\section{REFERENCES}

[1] G. Schreier, "Geometrical properties of SAR images," in SAR Geocoding: Data and Systems. Karlsruhe, Germany: Wichmann, 1993, pp. 103-134.

[2] U. Stilla, U. Soergel, and U. Thoennessen, "Potential and limits of InSAR data for building reconstruction in built-up areas," ISPRS J. Photogramm. Remote Sens., vol. 58, no. 1/2, pp. 113-123, Jun. 2003. 
[3] M. Quartulli and M. Datcu, "Stochastic geometrical modeling for built-up area understanding from a single SAR intensity image with meter resolution," IEEE Trans. Geosci. Remote Sens., vol. 42, no. 9, pp. 1996-2003, Sep. 2004

[4] U. Soergel, U. Thoennessen, A. Brenner, and U. Stilla, "High-resolution SAR data: New opportunities and challenges for the analysis of urban areas," Proc. Inst. Elect. Eng.-Radar, Sonar Navig., vol. 153, no. 3, pp. 294-300, Jun. 2006.

[5] F. Rottensteiner and C. Briese, "A new method for building extraction in urban areas from high-resolution LIDAR data," Int. Arch. Photogramm. Remote Sens. Spatial Inf. Sci., vol. 34, no. 3/A, pp. 295-301, 2002.

[6] M. Crespi et al., "Radiometric quality and DSM generation analysis of CartoSat-1 stereo imagery," in Proc. Int. Arch. Photogramm., Remote Sens. Spatial Inf. Sci., 2008, vol. 37, pp. 1349-1355.

[7] X. X. Zhu and R. Bamler, "Very high resolution spaceborne SAR tomography in urban environment," IEEE Trans. Geosci. Remote Sens., vol. 48, no. 12, pp. 4296-4308, Dec. 2010.

[8] S. Duque, C. Rossi, and T. Fritz, "Single-pass tomography with alternating bistatic TanDEM-X data," IEEE Geosci. Remote Sens. Lett., vol. 12, no. 2, pp. 409-413, Feb. 2015.

[9] A. Pauciullo, D. Reale, A. De Maio, and G. Fornaro, "Detection of double scatterers in SAR tomography," IEEE Trans. Geosci. Remote Sens., vol. 50, no. 9, pp. 3567-3586, Sep. 2012.

[10] C. Tison, F. Tupin, and H. Maitre, "A fusion scheme for joint retrieval of urban height map and classification from high-resolution interferometric SAR images," IEEE Trans. Geosci. Remote Sens., vol. 45, no. 2, pp. 496-505, Feb. 2007.

[11] E. Colin-Koeniguer and N. Trouv, "Performance of building height estimation using high-resolution PolInSAR images," IEEE Trans. Geosci. Remote Sens., vol. 52, no. 9, pp. 5870-5879, Sep. 2014.

[12] C. Rossi and S. Gernhardt, "Urban DEM generation, analysis and enhancements using TanDEM-X," ISPRS J. Photogramm. Remote Sens., vol. 85, pp. 120-131, Nov. 2013.

[13] D. Brunner, G. Lemoine, L. Bruzzone, and H. Greidanus, "Building height retrieval from VHR SAR imagery based on an iterative simulation and matching technique," IEEE Trans. Geosci. Remote Sens., vol. 48, no. 3, pp. 1487-1504, Mar. 2010.

[14] J. Tao, S. Auer, G. Palubinskas, P. Reinartz, and R. Bamler, "Automatic SAR simulation technique for object identification in complex urban scenarios," IEEE J. Sel. Topics Appl. Earth Observ. Remote Sens., vol. 7, no. 3, pp. 994-1003, Mar. 2014.

[15] R. Cossu, F. Dell'Acqua, D. Polli, and G. Rogolino, "SAR-Based seismic damage assessment in urban areas: Scaling down resolution, scaling up computational performance," IEEE J. Sel. Topics Appl. Earth Observ. Remote Sens., vol. 5, no. 4, pp. 1110-1117, Aug. 2012.

[16] W. Kropatsch and D. Strobl, "The generation of SAR layover and shadow maps from digital elevation models," IEEE Trans. Geosci. Remote Sens., vol. 28, no. 1, pp. 98-107, Jan. 1990.

[17] A. Thiele, J. Wegner, and U. Soergel, "Building reconstruction from multi-aspect InSAR data," in Radar Remote Sensing of Urban Areas ser. ser. Remote Sensing and Digital Image Processing, vol. 15, U. Soergel, Ed. Dordretch, The Netherlands: Springer-Verlag, 2010, pp. $187-214$.

[18] A. Thiele, M. M. Wurth, M. Even, and S. Hinz, "Extraction of builidng shape from TanDEM-X data," in Proc. ISPRS, 2013, vol. XL-1/W1, pp. $345-350$

[19] A. Monti Guarnieri, "SAR interferometry and statistical topography," IEEE Trans. Geosci. Remote Sens., vol. 40, no. 12, pp. 2567-2581, Dec. 2002.

[20] U. Spagnolini, "2-D phase unwrapping and instantaneous frequency estimation," IEEE Trans. Geosci. Remote Sens., vol. 33, no. 3, pp. 579-589, May 1995.

[21] M. Eineder, N. Adam, R. Bamler, N. Yague-Martinez, and H. Breit, "Spaceborne spotlight SAR interferometry with TerraSAR-X," IEEE Trans. Geosci. Remote Sens., vol. 47, no. 5, pp. 1524-1535, May 2009.
[22] R. Bamler and P. Hartl, "Synthetic aperture radar interferometry," Inv. Probl., vol. 14, no. 4, pp. R1-R54, Aug. 1998.

[23] D. Perissin and F. Rocca, "High-accuracy urban DEM using permanent scatterers," IEEE Trans. Geosci. Remote Sens., vol. 44, no. 11, pp. 3338-3347, Nov. 2006

[24] A. Thiele, E. Cadario, K. Schulz, U. Thonnessen, and U. Soergel, "Building recognition from multi-aspect high-resolution InSAR data in urban areas," IEEE Trans. Geosci. Remote Sens., vol. 45, no. 11, pp. 3583-3593, Nov. 2007.

[25] C. Prati and F. Rocca, "Limits to the resolution of elevation maps from stereo SAR images," Int. J. Remote Sens., vol. 11, no. 12, pp. 2215-2235, Dec. 1990.

[26] F. Gatelli et al., "The wavenumber shift in SAR interferometry," IEEE Trans. Geosci. Remote Sens., vol. 32, no. 4, pp. 855-865, Jul. 1994.

[27] O. Hellwich and H. Ebner, "Geocoding SAR interferograms by least squares adjustment," ISPRS J. Photogramm. Remote Sens., vol. 55, no. 4 , pp. $277-288$, Nov. 2000.

[28] M. Schwabisch, "A fast and efficient technique for SAR interferogram geocoding," in Proc. IEEE IGARSS, Jul. 1998, vol. 2, pp. 1100-1102.

[29] C. Oliver and S. Quegan, Understanding Synthetic Aperture Radar Images. Stevenage, U.K.: SciTech, 2004.

[30] C. Rossi, F. R. Gonzalez, T. Fritz, N. Yague-Martinez, and M. Eineder, “TanDEM-X calibrated raw DEM generation," ISPRS J. Photogramm. Remote Sens., vol. 73, pp. 12-20, Sep. 2012.

[31] G. Vasile, E. Trouve, J.-S. Lee, and V. Buzuloiu, "Intensity-driven adaptive-neighborhood technique for polarimetric and interferometric SAR parameters estimation," IEEE Trans. Geosci. Remote Sens., vol. 44, no. 6, pp. 1609-1621, Jun. 2006.

[32] S. Kay, Modern Spectral Estimation: Theory and Application. Englewood Cliffs, NJ, USA: Prentice-Hall, 1988.

[33] M. H. Hayes, Statistical Digital Signal Processing and Modeling. Hoboken, NJ, USA: Wiley, 1996, p. 460.

[34] T.-J. Shan, M. Wax, and T. Kailath, "On spatial smoothing for directionof-arrival estimation of coherent signals," IEEE Trans. Acoust., Speech, Signal Process., vol. 33, no. 4, pp. 806-811, Aug. 1985.

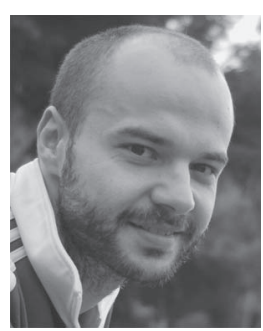

Cristian Rossi received the Laurea Magistrale (M.Sc.) degree in telecommunication engineering from Politecnico di Milano, Milan, Italy, in 2006.

From 2006 to 2008, he was with Aresys, Milan, Italy. Since 2008, he has been with the SAR Signal Processing Department, Earth Observation Center, German Aerospace Center (DLR), Oberpfaffenhofen, Germany, where he works on the development of the integrated TanDEM-X processor and on novel interferometry algorithms for synthetic aperture radar missions. His research interests include urban remote sensing, multisource data fusion, digital elevation models, and environmental parameter estimation.

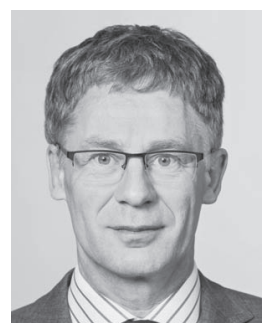

Michael Eineder (SM'01) is a specialist for synthetic aperture radar (SAR). He has been with the German Aerospace Center (DLR) since 1990, where he is currently heading the SAR Signal Processing Department of DLR's Remote Sensing Technology Institute. He has worked on a series of international SAR missions including the recent German missions TerraSAR-X and TanDEM-X. Since 2006, he has been a Part-Time Lecturer of remote sensing with the Technische Universitaet Muenchen (TUM), and since 2013, he has been a TUM Honorary Professor. His research interests encompass SAR, InSAR, and SAR imaging geodesy. 